\title{
Femtosecond Fiber Timing Distribution System for the Linac Coherent Light Source
}

\author{
Heng Li ${ }^{1,3,4}$, Lin-Jin Chen ${ }^{5}$, Haynes Pak Hay Cheng ${ }^{5}$, Justin E. May ${ }^{4}$, Steve Smith ${ }^{4}$, Kerstin Muehlig ${ }^{4}$, \\ Josef C. Frisch ${ }^{4}$, Alan R. Fry ${ }^{4}$, Franz X. Kärtner ${ }^{6,7}$, Philip H. Bucksbaum ${ }^{1,2,3,4}$ \\ ${ }^{1}$ Stanford PULSE Institute, ${ }^{2}$ Department of Physics, and ${ }^{3}$ Department of Applied Physics, Stanford University, Stanford, CA 94305, USA. ${ }^{4}$ SLAC \\ National Accelerator Laboratory, 2575 Sand Hill Road, Menlo Park, CA 94025, USA. Idesta Quantum Electronics, LLC, 56 Sparta Avenue, \\ Newton, NJ 07860, USA. ${ }^{6}$ Department of Electrical Engineering and Computer Science, and Research Laboratory of Electronics, Massachusetts \\ Institute of Technology, 77 Massachusetts Avenue, Cambridge, MA 02139, USA. ${ }^{7}$ Center for Free-electron Laser Science, DESY, and Physics \\ Department, University of Hamburg, Hamburg, Germany \\ Author e-mail address: liheng@slac.stanford.edu
}

\begin{abstract}
We present the design and progress towards implementation of a femtosecond fiber timing distribution system for the Linac Coherent Light Source at SLAC enabling machine diagnostic at the $10 \mathrm{fs}$ level.

OCIS codes: (060.2360) Fiber optics links and subsystems; (140.7240) UV, EUV, and X-ray lasers
\end{abstract}

The Linac Coherent Light Source (LCLS) at Stanford Linear Accelerator Center (SLAC) is the first hard x-ray freeelectron laser (FEL) of the world; it produces x-rays at wavelengths from 22 to $1.2 \AA$ with peak brightness ten orders of magnitude higher than traditional synchrotron sources [1]. Recent experiments have reported generation of approximately $3 \mathrm{fs} x$-ray pulses $[2,3]$. This opens the era of exploring the ultrafast dynamics of atoms, molecules, proteins and condensed matter [4-8] with time-resolved optical/x-ray pump-probe techniques. The temporal resolution of current experiments is limited by the timing jitter between optical and $\mathrm{x}$-ray pulses [9] generated from self-amplified spontaneous emission (SASE) process. An intrinsic timing jitter of approximately 120 fs root-meansquare (rms) with respect to the electron bunch arrival time at the end of the undulator has been reported [7]. Several approaches have been proposed to improve the timing of x-ray pulses. Recently, an x-ray/optical cross-correlator was implemented at LCLS that measures the relative jitter between the conventional and FEL $\mathrm{x}$-ray pulses and reduces the jitter to sub-25 fs rms in post analysis [10]. Furthermore, external seeding is expected to reduce the intrinsic timing jitter since the timing of the $\mathrm{x}$-rays would be governed by the timing of the seed within the electron bunch $[11,12]$, which would enable full synchronization of the optical and $\mathrm{x}$-ray pulses with sub-10 fs precision. Of such a technique, synchronization between seed and pump lasers would be implemented [13-16]. Preliminary test results of the key components for a 4 link system will be presented. Currently, the system is geared towards diagnostics to study the various sources of jitter at LCLS.

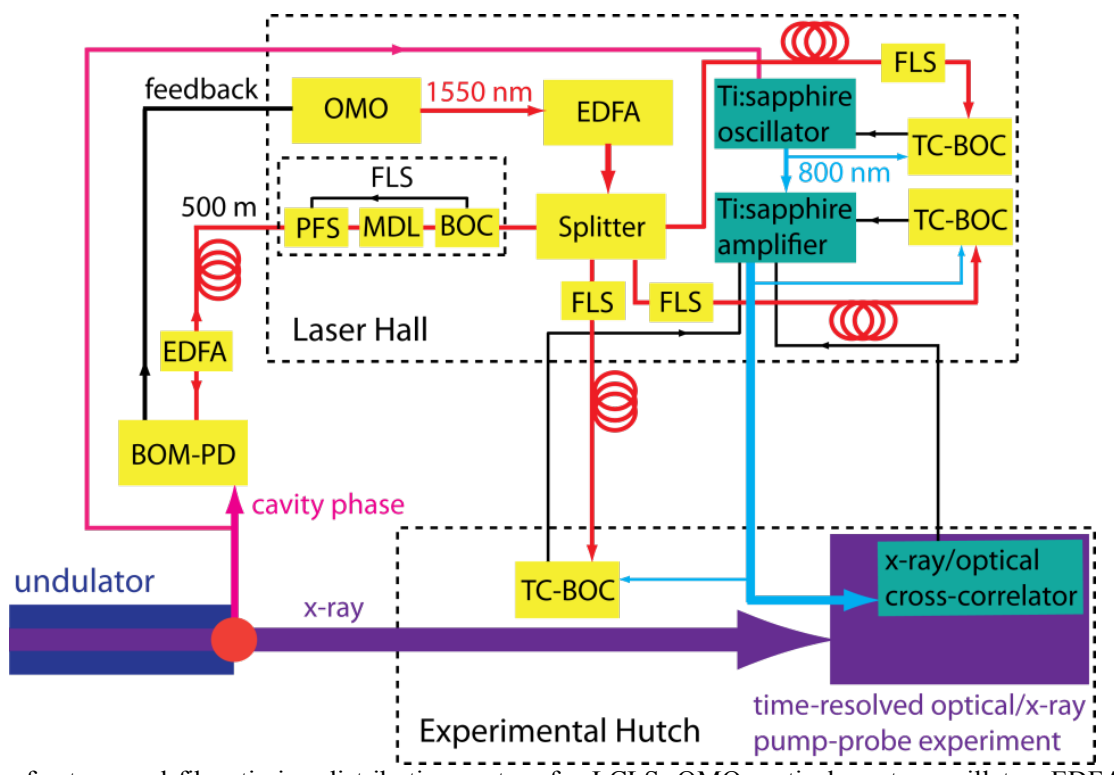

Fig.1. The layout of the femtosecond fiber timing distribution system for LCLS. OMOM, optical master oscillator; EDFA, Erbium-doped fiber amplifier; FLS, fiber link stabilizer; TC-BOC, two-color balanced optical cross-correlator; PFS, piezo-driven fiber stretcher; MDL, motorized delay line; BOM-PD, balanced-optical-microwave phase-detector. 
Figure. 1 shows the layout of the proposed femtosecond fiber-laser based timing system for LCLS. In the design, a $68 \mathrm{MHz}$ Er-doped fiber laser (Toptica FErb 1560) located in the laser hall is used as an optical master oscillator (OMO) synchronized to the rf-clock signal at $476 \mathrm{MHz}$ [13]. Since the rf pick-up cavity is located at the end of the undulator, where access is highly restricted, the pulse train from the OMO is delivered to the rf pick-up location by a dispersion-compensated optical fiber link stabilized with a single-crystal PPKTP balanced optical cross-correlator (BOC) which provides a timing sensitivity of $4.8 \mathrm{mV} / \mathrm{fs}$ with a $\sim 500 \mathrm{fs}$ linear region as shown in Fig. 2(a). Preliminary results show a rms timing jitter of $13-\mathrm{fs}$ after stabilization. The performance is currently limited by the resonance of piezo fiber stretcher at around $18 \mathrm{kHz}$ which can be further reduced by improved feedback electronics. The optical and rf timing is achieved with a balanced optical-microwave phase detector [13], and the baseband phase error signal synchronizes the OMO to the rf reference (RMO). Figure 2(b) shows the phase noise spectrum of the OMO when free-running and locked to the RMO measured with the same photodetector, which shows tight synchronization within the locking bandwidth of around $1 \mathrm{kHz}$. The pulses from the synchronized OMO are then distributed via stabilized optical fiber links to provide the timing reference for the Ti:sapphire oscillator and amplifier in the laser room. Optical-optical synchronization between the delivered synchronization pulses by the fiber link and the Ti:Sapphire laser is achieved by a two-color balanced optical cross-correlator (TC-BOC) based on type I sum-frequency generation between $800 \mathrm{~nm}$ and $1560 \mathrm{~nm}$ pulses [16]. Fig. 2(c) shows the obtained S-curve generated with a Ti:sapphire oscillator and OMO, which shows a timing sensitivity of $\sim 50 \mathrm{mV} / \mathrm{fs}$ (Fig. 2(c)). The Ti:sapphire oscillator was pre-synchronized to the RMO with a traditional rf lock and achieved a typical rms timing jitter of $\sim 150 \mathrm{fs}$, which is beyond the current capture range of the TC-BOC $(\sim 120 \mathrm{fs})$. Work on improving the capturing range of the TC-BOC as well as optimizing the rf pre-locking of the Ti:sapphire laser to make the transition from rf locking to TC-BOC locking and achieve better optical-optical synchronization is in progress.
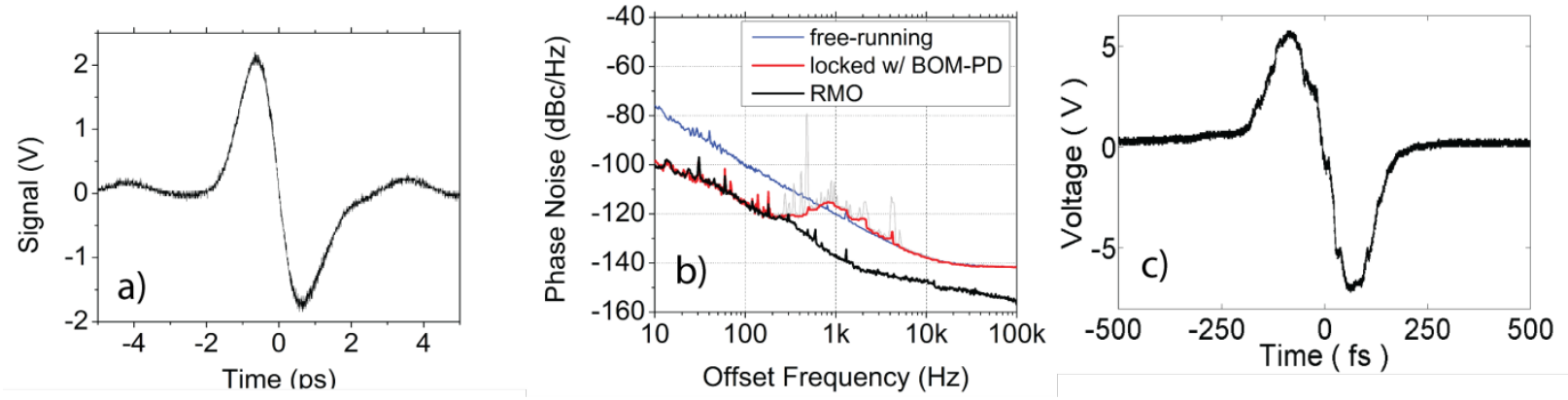

Fig.2. The timing system measurements. a) S-curve from the balanced optical cross-correlator in stabilized fiber timing link; b) phase noise spectrum of OMO when free-running and locked to the rf reference; c) the S-curve of the TC-BOC.

In conclusion, we report on progress towards a femtosecond fiber-laser based timing distribution system at LCLS. The performance of major components for optical-microwave, optical-optical synchronization modules, and optical timing distribution in a fully operating $\mathrm{x}$-ray FEL is presented.

[1] P. Emma et al., "First lasing and operation of an ångstrom-wavelength free-electron laser," Nat. Photonics 4, 641-647 (2010).

[2] Y. Ding et al., "Measurements and Simulations of Ultralow Emittance and Ultrashort Electron Beams in the Linac Coherent Light Source," Phys. Rev. Lett. 102, 254801 (2009).

[3] Y. Ding et al., "Femtosecond X-Ray Pulse Characterization in Free-Electron Lasers Using a Cross-Correlation Technique," Phys. Rev. Lett. 109, $254802(2012)$.

[4] A. H. Zewail, "Femtochemistry: Atomic-Scale Dynamics of the Chemical Bond," J. Phys. Chem. A, 104, 5660 (2000).

[5] J. P. Cryan et al., "Auger Electron Angular Distribution of Double Core-Hole States in the Molecular Reference Frame," Phys. Rev. Lett., 105, 083004 (2010).

[6] V. S. Petrović et al., "Transient X-Ray Fragmentation: Probing a Prototypical Photoinduced Ring Opening,” Phys. Rev. Lett., 108, 253006 (2012).

[7] J. M. Glownia et al., "Time-resolved pump-probe experiments at the LCLS,” Opt. Express, 18, 17620 (2010).

[8] M. Trigo et al., "Direct measurement of time-dependent density-density correlations in a solid through the acoustic analog of the dynamical Casimir effect," submitted to Nature, 2013.

[9] F. Tavella et al., "Few-femtosecond timing at fourth-generation X-ray light sources," Nat. Photonics 5, 162- 165 (2011).

[10] M. R. Biont et al., "Spectral encoding of x-ray/optical relative delay," Opt. Express, 19, 21855-21865 (2011).

[11] G. Stupakov, "Using the Beam-Echo Effect for Generation of Short-Wavelength Radiation," Phys. Rev. Lett. , 102, 074801 (2009).

[12] D. Xiang et al., "Evidence of High Harmonics from Echo-Enabled Harmonic Generation for Seeding X-Ray Free Electron Lasers," Phys.

Rev. Lett. , 108, 024802 (2012)

[13] J. Kim, et al., "Balanced optical-microwave phase detectors for optoelectronic phase-locked loops," Opt. Letters, 31, 3659 (2006).

[14] J. Kim, et al., "Long-term femtosecond timing link stabilization using a single-crystal balanced cross correlator," Opt. Lett,, 32, 1044 (2007)

[15] J.Kim, et al., "Drift-free femtosecond timing synchronization of remote optical and microwave sources," Nat. Photonics 2, 733-736 (2008).

[16] S. Schulz, et al., "An Optical Cross-Correlation Scheme to Synchronize Distributed Laser Systems at FLASH," 11th European Particle Accelerator Conference, Genoa, Italy, 23 - 27 Jun 2008, pp.THPC160. 